\title{
Prise en compte de la durabilité dans la représentation sociale de la croissance économique.
}

Sustainability in the social representation of growth.

\section{Hélène Blanc et Adeline Raymond}

\section{OpenEdition}

Journals

Édition électronique

URL : http://journals.openedition.org/developpementdurable/9069

DOI : 10.4000/developpementdurable.9069

ISSN : 1772-9971

Éditeur

Association DD\&T

Référence électronique

Hélène Blanc et Adeline Raymond, «Prise en compte de la durabilité dans la représentation sociale de la croissance économique. », Développement durable et territoires [En ligne], Vol. 2, n 3 | Décembre 2011, mis en ligne le 03 décembre 2011, consulté le 19 avril 2019. URL : http://

journals.openedition.org/developpementdurable/9069; DOI : 10.4000/developpementdurable.9069

Ce document a été généré automatiquement le 19 avril 2019.

Licence Creative Commons

Développement Durable et Territoires est mis à disposition selon les termes de la licence Creative Commons Attribution - Pas d'Utilisation Commerciale 4.0 International. 


\title{
Prise en compte de la durabilité dans la représentation sociale de la croissance économique.
}

\author{
Sustainability in the social representation of growth.
}

Hélène Blanc et Adeline Raymond

1 Cette contribution s'inscrit dans une étude plus large centrée sur les représentations sociales des concepts de croissance et de décroissance économiques dont l'objectif est d'une part, d'étudier comment des concepts théoriques sont appropriés par le sens commun et d'autre part, d'observer l'intégration éventuelle d'une idée nouvelle, la décroissance. Dans ce but, des mesures sont réalisées tous les deux ans (2004, 2006, 2008 ; l'exploitation des résultats 2010 est en cours). L'approche est pluridisciplinaire, mobilisant le champ de la science économique et celui de la psychologie sociale et plus particulièrement la théorie des représentations sociales.

2 À partir de cette étude bisannuelle centrée sur les concepts de "croissance", de "décroissance » et de "richesse ", la question du sens de la notion de «développement durable » nous a semblé pertinente. En effet, l'omniprésence de cette thématique dans les discours des divers acteurs, qu'ils soient politiques, économiques, de la société civile, etc., conduit à mettre l'accent sur la dimension de soutenabilité des activités humaines: les actes de production ou de consommation deviennent aujourd'hui «durables». Outre l'effet de mode, la prégnance de cette idée de durabilité, ou de soutenabilité, montrerait l'importance que prennent aujourd'hui les considérations écologiques.

On peut ainsi analyser la façon dont les individus intègrent cette dimension de durabilité lorsqu'ils sont interrogés sur le concept de croissance économique (et de manière connexe sur la décroissance et la richesse). Remettent-ils en question l'idée d'une croissance économique infinie? La conditionnent-ils à des prises en compte de contraintes environnementales? La conçoivent-ils comme une "autre croissance", « verte », « écologique » ou " durable»? Dans un premier temps, nous clarifierons les termes de croissance et de développement durable afin de montrer le lien qui les unit. 
Nous poserons ensuite le cadre théorique choisi pour étudier les représentations sociales puis nous exposerons nos méthode et résultats centraux.

\section{Croissance et développement durable. Les concepts}

4 Même si la notion de développement durable préexistait à la Commission Mondiale sur l'Environnement et le Développement (CMED créé par l'ONU en 1983), la définition généralement retenue émane du rapport Brundtland qui le caractérise comme «le développement qui répond aux besoins du présent sans compromettre la capacité des générations futures à répondre aux leurs» (CMED, 1987, p. 51). Ceci n'est cependant qu'une des nombreuses formulations fournies par ce rapport... La traduction française de cette notion (sustainable development) hésite entre «développement soutenable» et " développement durable ». C'est néanmoins cette seconde option qui paraît aujourd'hui la plus utilisée.

\subsection{Croissance et développement : deux notions liées}

5 Malgré l'apparente tautologie du propos, le développement durable renvoie d'abord à la notion de développement économique. Longtemps, pour les économistes, le développement a été assimilé aux différentes étapes de la croissance, par analogie avec le développement de l'individu passant de l'enfance à l'adolescence puis à l'âge adulte... C'est notamment la perspective de Rostow (1960) présentant le développement comme un processus de croissance auto-entretenue à travers cinq phases successives: la société traditionnelle, les conditions préalables au démarrage, le démarrage lui-même, la marche vers la maturité, l'ère de la consommation de masse.

Cependant, la contribution fondamentale de Perroux (1961) a remis en cause cette synonimie entre croissance et développement. Pour cet économiste, la croissance renvoie à l'augmentation quantitative des productions de biens et services créées sur un territoire donné pendant un an. Elle est évaluée par le Produit Intérieur Brut, somme des valeurs ajoutées des agents résidents. Le développement, quant à lui, renvoie à des transformations qualitatives plus complexes: politiques, culturelles, institutionnelles, améliorations des systèmes de santé et d'éducation... Il est donc généralement lié à une idée de " progrès » et de « bien-être » des populations concernées.

7 À la suite de ces deux auteurs, les économistes prennent ainsi garde à ne pas confondre croissance et développement. Il peut d'ailleurs exister ce que le Programme des Nations Unies pour le Développement (PNUD, 1996) a qualifié de «mauvaise croissance », c'est-àdire une croissance sans création d'emplois, sans résorption des inégalités, sans progrès vers la démocratie, avec perte de l'identité culturelle ou avec épuisement des ressources naturelles... autrement dit, une croissance économique sans développement. Cependant, Perroux lorsqu'il insistait sur cette distinction essentielle, estimait que le développement accompagnait la croissance. Il permet la croissance tout en en étant la conséquence : « Le développement est la combinaison des changements mentaux et sociaux d'une population qui la rendent apte à faire croitre, cumulativement et durablement, son produit réel global » (Perroux, 1961, cité par Vivien, 2005, p. 63). Cette complémentarité entre croissance et développement est, en outre illustrée, par l'indicateur synthétique créé pour mesurer ce dernier: l'Indicateur de Développement Humain (IDH) qui prend en compte trois éléments: le PIB/habitant mesurant le niveau de vie; l'espérance de vie et des 
indicateurs d'éducation (taux de scolarisation et taux d'alphabétisation). La croissance n'est plus considérée comme une condition nécessaire et suffisante au développement mais ce dernier suppose néanmoins la croissance. Le développement durable est d'abord un développement, donc une croissance, qui peut perdurer dans le temps.

Le rapport Brundtland intègre cette notion de développement, dépassant une simple croissance économique. C'est, nous disent ses rédacteurs, une croissance économique qui bénéficie à l'ensemble de la société puisqu'elle permet une meilleure répartition des revenus. Or, les travaux du CMED intègrent une autre dimension: celle d'un développement écologiquement soutenable. Ainsi trois piliers sont mis en avant dans le rapport: économique; social et environnemental. C'est bien cette dimension environnementale qui constitue la véritable nouveauté dans la notion de « développement durable ».

9 Cependant, un rapide aperçu de son histoire (Flipo, 2007) montre une évolution notable de l'acception de ce terme. Les premiers questionnements sont d'abord théoriques et portent sur une critique forte de la croissance des «trente glorieuses». Les travaux critiques de la société de consommation sont nombreux : Illich, Partant, Gorz, Ellul,... Évoquons aussi Georgescu-Roegen dans les années 1960 qui introduit l'idée de décroissance, insistant sur l'impossibilité d'une croissance illimitée de la production dans un monde où les ressources sont limitées (Georgescu-Roegen, 1979). Une autre contribution va jouer un rôle de premier plan : le rapport du Club de Rome, dit également rapport Meadows, Limits to Growth (qui sera traduit par Halte à la croissance) en 1972.

Après ces réflexions théoriques, des négociations internationales sur ces thématiques sont menées au sein de l'ONU. Lancées lors de la Conférence internationale sur l'environnement en 1972 à Stockholm, où il est question d'« écodéveloppement », la Commission Mondiale sur l'Environnement et le Développement est créée en 1983. Cette dernière rédige le célèbre rapport Brundtland et définit le développement durable. Ainsi, cette notion est d'abord forgée dans ces grandes conférences à la recherche de compromis acceptables par tous : pays développés et pays en développement; syndicats patronaux et salariés; recevabilité des opinions publiques... Il s'opère un certain glissement sémantique : la dimension économique prenant de plus en plus d'importance. Plutôt qu'un concept scientifique, solidement établi, «le développement durable est un concept diplomatique qui cache des divergences importantes et des enjeux géopolitiques majeurs ", comme le souligne Flipo (2007, p. 60). Dés son origine, cette notion de développement durable a cristallisé les tensions entre ces deux pôles : économique et environnemental.

\subsection{Permanence d'un débat sur la nature de ces liens}

11 Depuis ses origines dans les années 1970, les réflexions autour de l'intégration de cette dimension environnementale dans l'idée de développement a fait naître des débats et des crispations. Le rapport Meadows a suscité, par exemple, des réactions très vives tant de la part des syndicats de salariés que du syndicat patronal, le CNPF à l'époque, qui y voyait une remise en cause des règles fondamentales de notre économie...

12 Aujourd'hui, le débat reste vif autour de la notion de développement durable. Trop consensuelle, pour les uns, elle minimise finalement la dimension de soutenabilité écologique qu'elle est censée porter. C'est l'avis des tenants de la décroissance, qui estiment que cette expression est un oxymore, le développement ne rompant pas avec le « dogme de la croissance » (Latouche, 2001). Pour d'autres, en revanche, l'introduction de 
ce terme tire son utilité précisément de cette origine consensuelle. Elle permet de poser les questions pertinentes et de s'interroger sur le contenu, la qualité, de la croissance: une " autre croissance ", une croissance «verte ", intégrant la nécessaire protection de notre environnement serait possible (Haribey, 2004).

Notre objet n'est pas ici de trancher ce débat mais de nous borner à constater que l'idée même de développement durable implique un questionnement sur le concept de croissance. Elle pose les limites d'une croissance productiviste entraînant des effets négatifs sur nos écosystèmes. Elle appelle un changement de cap, vers l'arrêt d'une croissance infinie ou, pour d'autres, vers une « autre croissance ».

En tout état de cause, nous pouvons émettre l'hypothèse que le discours actuellement omniprésent sur la durabilité puisse conduire à une inflexion dans les représentations sociales de la croissance. L'importance de cette thématique conduirait à une connotation plus négative du concept de croissance, synonyme non plus seulement de richesses, mais également de dégradations des écosystèmes. Les groupes sociaux intègreraient aujourd'hui ces considérations écologiques quand cette notion est évoquée. Le cadre d'analyse que représente l'étude des représentations sociales peut nous permettre de répondre à ces interrogations.

\section{Le cadre théorique : la théorie des représentations sociales}

15 Moscovici (1961/1976) présente cette théorie lors de sa recherche sur la représentation de la psychanalyse. L'auteur voulait d'étudier l'intégration progressive d'une théorie scientifique dans la pensée de sens commun. Dans ce travail, il décrit les représentations sociales comme des réalités partagées constituant un mode spécifique de connaissances et de communication socialement construites. Cette transmission et ce partage permettent aux individus d'agir à un moment donné. Elles deviennent ainsi le «sens commun » avec un caractère d'évidence dû à l'effet de consensus. C'est un peu la traduction du « on sait bien que... ", pensée quotidienne ou naïve qui justifie nos conduites. Sans aller jusqu'à déterminer une limite entre pensées scientifique et naïve, la théorie des représentations sociales permet précisément de mesurer ce décalage ou cette appropriation de concepts et de notions dites « scientifiques » par les groupes sociaux. Il s'agit de savoir comment « le jeu de la science devient en partie le jeu du sens commun » (Moscovici et Hewstone, 2003, p. 546) et l'éclaircissement passe par la théorie des représentations sociales.

Moscovici propose un modèle décrivant deux processus qui président à l'élaboration de la représentation sociale. Le premier, l'objectivation, consiste à passer de l'abstrait au concret. Il s'agit tout d'abord d'une sélection, par le groupe concerné, des informations relatives à l'objet de représentation, car toutes ne peuvent pas être conservées. Certaines, qui ne sont pas en cohérence avec les normes et les valeurs du groupe seront simplement rejetées, d'autres subiront des modifications. Ensuite, ces informations seront intégrées au discours du groupe élaborant un noyau figuratif, c'est-à-dire une schématisation de l'objet initial. Le noyau figuratif, une fois constitué, devient une expression directe de cette théorie : il est simple, concret et fait référence à l'ensemble des normes et valeurs du groupe. Le second processus, l'ancrage, consiste à placer, dans un cadre de référence familier, le nouvel objet schématisé. L'objectif visé est qu'il devienne un système d'interprétation générant ses propres catégories d'explication. La représentation ainsi 
constituée est alors fonctionnelle parce qu'elle se réfère aux valeurs du groupe ; celui-ci pouvant pratiquement l'utiliser dans son quotidien et dans ses nombreuses interactions avec son environnement. Grâce à ce cadre théorique, il devient possible d'observer la possible prise en compte considérations écologiques dans la représentation sociale de la croissance économique.

\section{Contributions à une étude des représentations sociales de la croissance, de la décroissance et de la richesse}

17 Comme nous l'avons évoqué en introduction, ce travail s'inscrit dans une étude plus large portant sur l'évolution des représentations sociales de la croissance, de la décroissance et de la richesse. Ainsi depuis 2004, avec un rythme bisannuel, nous recueillons, lors de la passation d'un questionnaire d'enquête, des données permettant de mesurer l'évolution de ces représentations. Ce questionnaire propose en premier lieu des questions d'évocation formulées ainsi : "À quoi vous fait penser l'expression "Croissance économique »?, Écrivez ci-dessous, les mots ou expressions (entre 3 et 10) qui vous viennent à l'esprit. » Les termes de « décroissance économique » et « richesses » sont aussi objet de question d'évocation et seront traités, ici, de manière connexe.

L'analyse des évocations permet de connaître la fréquence d'apparition des termes ainsi que le numéro d'ordre (le rang). Cette approche postule qu'un mot apparaissant fréquemment et en début d'évocation revêt un caractère central dans la représentation. En effet, les travaux d'Abric $(1992,1994)$ reprennent le modèle de noyau figuratif pour aboutir à celui de noyau central. L'auteur y développe le concept de représentations sociales en s'attachant à la mise en évidence d'une structure hiérarchisée d'éléments cognitifs. Les représentations sociales sont alors définies comme deux systèmes à la fois spécifiques et complémentaires : le "système central ", fondement stable et rigide de la représentation, et le «système périphérique " plus mouvant puisque déterminé par les caractéristiques individuelles des individus du groupe. Les questions d'évocations nous permettent donc de définir les éléments appartenant au noyau central de la représentation sociale. Cette analyse est rendue possible grâce à la méthode créée par Vergès (1992) et reposant d'une part sur une analyse lexicographique et d'autre part sur la définition de catégories. Ce traitement s'effectue à l'aide du logiciel Evoc2000 (version 2002).

Une appréciation par le sujet de la connotation des termes "croissance » et "décroissance» est ensuite demandée, déclinée en trois points: négative/neutre/ positive. Les personnes sont aussi interrogées sur le terme "richesses » non repris ici. Pour le recueil de 2008 , nous avons ajouté une question concernant la connotation du terme « développement durable ».

Les représentations sociales étant intimement liées au contexte dans lequel elles se développent et aux caractéristiques des groupes qui les portent, nous avons pris en compte un certain nombre de variables qui nous paraissent pertinentes. En effet, une série de questions concerne les caractéristiques du répondant (sexe, âge, études, situation familiale, nombre d'enfants), ainsi que deux échelles d'attitude en cinq points: l'une portant sur le positionnement politique (extrême droite/droite/centre/gauche/extrême gauche) et l'autre permettant de mesurer un indice de proximité vis-à-vis du courant 
écologiste (tout à fait proche/plutôt proche/ni proche, ni éloigné(e)/plutôt éloigné(e)/ tout à fait éloigné(é)).

21 Nous avons donc procédé à trois recueils de données : 2004, 2006 et 2008. Chaque passation a été réalisée auprès de plus de 400 étudiants en $1^{\text {er }}$ cycle universitaire : DUT Génie Biologique, L1/L2 de Psychologie et DUT tertiaire. En effet, même si notre échantillon est uniquement constitué d'étudiants, nous avons souhaité diversifier leurs origines. Certains ont eu durant leurs études des enseignements d'économie (DUT tertiaire) mais ce n'est généralement pas le cas pour les autres, les plus nombreux. Enfin, afin d'éviter que les individus définissent un terme en fonction de l'autre, ces échantillons ont été divisés en deux: la moitié a été interrogée sur la "croissance économique " (2004: n=255; $2006: \mathrm{n}=218$ et $2008: \mathrm{n}=207)$ et l'autre sur la « décroissance économique ».

\section{Appréciations : caractériser la croissance}

\subsection{Les connotations : le caractère positif de la croissance}

Le tableau ci-dessous présente les résultats obtenus pour les questions portant sur les connotations.

Tableau 1. Présentation, pour les termes soumis à la connotation, des effectifs et des pourcentages en fonction de la nature de celle-ci.

\begin{tabular}{|c|c|c|c|c|c|c|c|}
\hline Nature de la connotation & \multicolumn{2}{|c|}{ Négative } & \multicolumn{2}{|c|}{ Neutre } & \multicolumn{2}{|c|}{ Positive } & \\
\hline Termes connotés & effectif & $\%$ & effectif & $\%$ & effectif & $\%$ & Total \\
\hline «Développement dural & 3 & 0,73 & 66 & 16,02 & 343 & 83,25 & 412 \\
\hline «Croissance» - 2004, 2 & 66 & 7,48 & 156 & 17,69 & 660 & 74,83 & 882 \\
\hline "Décroissance» - 2004, 2006 et 2008 & 749 & 85.60 & 98 & 11.20 & 28 & 3.20 & 875 \\
\hline
\end{tabular}

23 Sans équivoque aucune, le terme de " développement durable » est connoté positivement. À peine $1 \%$ des répondants l'envisage négativement. Il ne fait donc aucun doute que le développement durable est perçu comme un phénomène favorable. Le terme «croissance» est aussi jugé positivement et ce, sans différence statistiquement significative entre les trois recueils de données, permettant donc une présentation commune. La croissance est une notion socialement valorisée et nous verrons pourquoi dans l'analyse des questions d'évocations. Ces résultats sont confortés par la connotation du terme décroissance jugée à $85 \%$ négative sans distinction d'année. Le caractère positif de la croissance se maintient donc sans évolution visible.

\subsection{Les évocations : stabilité de la représentation sociale de la croissance}

La méthode des évocations de Vergès permet donc de produire des hypothèses de centralité et de déterminer ainsi les éléments susceptibles d'appartenir au noyau central par leur fréquence élevée et leur rang d'apparition faible. Le tableau, ci-après, reprend les éléments susceptibles d'appartenir système central. 
Tableau 2. Présentation des termes les plus souvent cités en début d'évocation de la « croissance économique » en fonction de l'année de passation.

\begin{tabular}{|l|c|c|c|c|c|c|c|c|}
\hline \multicolumn{2}{|c|}{ "Croissance 2004 » } & \multicolumn{2}{c|}{ «Croissance 2006 » } & \multicolumn{3}{c|}{ "Croissance 2008 » } \\
\hline \multicolumn{1}{|c|}{ Mots } & effectif & $\%$ & Mots & effectif & $\%$ & Mot & effectif & $\%$ \\
\hline Augmentation & 90 & 35,29 & Augmentation & 67 & 30,73 & Augmentation & 64 & 30,92 \\
\hline Développement & 62 & 24,31 & Développement & 58 & 26,61 & Développement & 52 & 25,12 \\
\hline PIB & 52 & 20,39 & PIB & 44 & 20,18 & PIB & 40 & 19,32 \\
\hline Richesse & 59 & 23,14 & Richesse & 49 & 22,48 & Richesse & 38 & 18,36 \\
\hline
\end{tabular}

Quelle que soit l'année de passation du questionnaire, il y a une hypothèse d'appartenance au système central de la représentation sociale de la croissance pour les mots : augmentation, développement, PIB, et richesses. Cela paraît en adéquation avec la définition donnée par les économistes qui conçoivent la croissance économique comme l'augmentation des richesses au niveau national, évalué par le PIB, et facteur de développement. Les résultats aux questions d'évocations renvoient au niveau de vie dont l'augmentation entraîne plus de bien-être. C'est donc bien une notion socialement valorisée. L'analyse approfondie en termes de catégories ne fait que conforter ce résultat : la croissance économique renvoie aux idées de production de richesses; d'augmentation du volume de l'emploi et des revenus distribués; d'élévation du niveau de vie et en définitive l'amélioration du bien-être. Elle permet le développement du pays.

De plus, aucun des différents critères retenus (sexe, type d'études, orientation politiques et proximité avec le courant écologiste) n'agit significativement sur le système central. Il existe donc une représentation sociale de la croissance économique commune chez les étudiants de notre échantillon en cohérence avec la définition donnée par les économistes. L'enracinement de ce concept dans notre société justifie ce consensus qui ne connaît aucune évolution depuis 2004. Cette stabilité n'est en rien infléchie par l'introduction dans le discours de la notion de durabilité. Notre hypothèse d'une modification de la représentation sociale de la croissance est donc infirmée. L'idée de croissance semble ancrée, intégrée comme une norme, une valeur fondatrice pour notre échantillon. Ce constat pourrait être certainement vérifié sur l'ensemble de notre société. Nous allons tenter de savoir si, malgré cette stabilité, il existe une percée ou prise en considération d'élément référents à l'écologie dans les structures périphériques des représentations sociales.

\subsection{Absence de considérations écologiques}

Pour ce faire, nous avons étudié les mots cités et leur fréquence d'apparition pour les termes soumis à l'évocation de la "croissance économique». Nous avons élargi aux résultats obtenus à celle de "richesses" (notion plus large mais liée) et celle de "décroissance» (notion critique). En effet, il est des mots ou expressions, même marginaux, dont nous pourrions observer l'émergence, grâce aux recueils récurrents de données. Le tableau 3 reprend certains termes que nous avons choisis pour leur proximité sémantique avec la notion de développement durable. 
Tableau 3. Nombre de mots cités par année en fonction de l'évocation.

\begin{tabular}{|l|c|c|c|c|c|c|c|c|c|}
\cline { 2 - 10 } \multicolumn{1}{c|}{} & \multicolumn{3}{c|}{2004} & \multicolumn{3}{c|}{2006} & \multicolumn{3}{c|}{2008} \\
\cline { 2 - 11 } \multicolumn{1}{c|}{} & $\mathrm{C}$ & $\mathrm{D}$ & $\mathrm{R}$ & $\mathrm{C}$ & $\mathrm{D}$ & $\mathrm{R}$ & $\mathrm{C}$ & $\mathrm{D}$ & $\mathrm{R}$ \\
\hline Développement durable & 1 & & & 1 & 1 & 1 & 1 & & 1 \\
\hline Soutenable / Soutenabilité & & & & & & & & & \\
\hline Ecologie / écologique & 1 & & & 1 & 1 & & & 1 & \\
\hline Climat / climatique & & & & & & & & & \\
\hline Environnement - al - e & & 1 & 1 & & & 5 & 1 & 1 & 2 \\
\hline Pollution & & & 1 & 4 & 1 & & 4 & 2 & 1 \\
\hline Gaspillage & & & 2 & & & 2 & & & 2 \\
\hline Effet de serre & & & & & 1 & & & & \\
\hline Energies & & & 1 & & & 1 & & & 3 \\
\hline Renouvelable & & & & & & & & & \\
\hline Biologique / bio & & & & & & & & 2 & \\
\hline Biodiversité & & & & & & 1 & & & \\
\hline Nombre total de mots cités & 1884 & 1848 & 3244 & 1407 & 1404 & 2544 & 1244 & 1326 & 2254 \\
\hline
\end{tabular}

$C=$ Croissance $; D=$ décroissance $; R=$ Richesse

Les résultats sont clairs : la prise en compte des considérations écologiques est quasiinexistante quand les répondants évoquent "la croissance ", "la décroissance » ou encore la « richesse ». Certes, le nombre d'évocations connait une augmentation en valeur absolue (de 8 en 2004 à 21 en 2008) mais reste toujours en dessous de $0,5 \%$ du total des mots cités. Aucun des termes choisis ne déroge à la règle.

Il est, par exemple, assez étonnant que le mot « climat » n'apparaisse jamais alors que les discours actuels insistent particulièrement sur la question du " changement climatique ", montrant même un certain glissement sémantique en défaveur de la notion développement durable. L'hypothèse que l'importance du développement durable dans le discours ambiant entraînerait une modification de la représentation sociale de la croissance, en intégrant la dimension de durabilité, est infirmée. Cela rejoint la thèse de Moscovici selon laquelle seraient rejetées les informations rentrant en contradiction avec les normes et valeurs du groupe dans l'élaboration de la représentation sociale. Ainsi toutes les considérations écologiques semblent exclues, pour l'heure, comme si elles remettaient en cause l'idée même de croissance.

\section{Conclusion}

Nos recueils de données montrent une stabilité notable des éléments susceptibles d'appartenir aux noyaux centraux des représentations sociales de la croissance. Aucune considération écologique ne vient remettre en question ces représentations. Plus encore, les systèmes périphériques ne semblent pas connaitre de modification. Ceci infirme nos hypothèses de départ. Cette non-prise en compte des considérations écologiques confirme l'ambiguïté de la notion de développement durable déjà évoquée : large et consensuelle, elle est très bien acceptée. Au prix, cependant, d'une certaine perte de contenu puisqu'elle n'entraîne aucune remise en cause de la connotation positive de la croissance, et ne conduit pas à l'évocation d'une "autre " croissance. Peut-être que l'importance médiatique que revêt aujourd'hui cette idée de durabilité, avec en particulier l'impact du Grenelle de l'environnement ou l'écho des grandes conférences internationales comme celle de l'ONU à Copenhague en décembre 2009, conduiront à une inflexion lors de la prochaine passation de questionnaire en 2010. 


\section{BIBLIOGRAPHIE}

Abric J. C., 1992, «Système central, système périphérique : leurs fonctions et leur rôle dans la dynamique des représentations sociales ", Première Conférence Internationale sur les Représentations Sociales, Ravello, Italie.

Abric J. C., 1994, « Les représentations sociales : aspects théoriques », in Abric J.C. (dir.). Pratiques sociales et représentations, Paris : PUF, p. 11-35.

Club de Rome, 1972, The Limits to Growth, New-York : Universe Books.

CMED, 1987, Notre avenir à tous, (traduction française), Montréal : Éditions du Fleuve.

Flipo F., 2007, Le Développement durable, Paris : Bréal.

Georgescu-Roegen N., 1979, La Décroissance. Entropie, écologie, économie, Paris : Le Sang de la Terre. (3 $3^{\text {ème }}$ édition, 2006).

Haribey J-M., 2004, « Développement ne rime pas forcément avec croissance », Le Monde

Diplomatique, juillet.

Latouche S., 2001, « En finir une fois pour toutes avec le développement », Le Monde Diplomatique, mai.

Moscovici S. \& Hewstone M., 2003, « De la science au sens commun » in Moscovici S. (dir.),

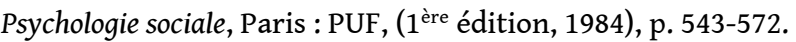

Moscovici S., 1976, La psychanalyse, son image et son public, Paris : PUF, (1 ère édition, 1961).

Perroux F., 1961, L'Économie du XXè siècle, Paris : PUF.

PNUD, 1996, Rapport sur le développement humain 1996.

Rostow W. W., 1960, Les étapes de la croissance économique, Paris : Le Seuil (traduction française $2^{\text {nde }}$ édition : 1970)

Vergès P., 1992, «L'évocation de l'argent : une méthode pour la définition du noyau central d'une représentation », Bulletin de psychologie, XLV, 405, p. 203-209.

Vivien F-D., 2005, Le développement soutenable, Paris : La Découverte.

\section{RÉSUMÉS}

Cette contribution porte sur l'évolution de la représentation sociale de la croissance économique permettant l'étude de l'appropriation de ce concept scientifique par le sens commun. L'omniprésence actuelle de la thématique du «développement durable » dans les discours ambiants conduit à mettre l'accent sur la dimension de soutenabilité des activités humaines. On pourrait par conséquent s'attendre à une prise en compte des considérations écologiques dans les manières de produire et de consommer, ce qui pourrait entraîner une inflexion dans la représentation sociale de la croissance économique. Les résultats issus de données recueillies auprès d'étudiants en 2004, 2006 et 2008 infirment notre hypothèse et présentent une forte stabilité de la représentation : aucune considération écologique n'apparaît. L'idée de croissance semble intégrée comme une valeur fondatrice, immuable. 
This contribution analyses the social representation of economic growth and its evolution. The aim is to study how common sense appropriates scientific concepts. The expression of sustainable development is nowadays frequently present in the public debate, focusing attention on the sustainable dimension of human activities. We should therefore expect an integration of these ecological issues in consumption and production modes and consequently a modification of the social representation of economic growth. About 1200 students were questioned in 2004, 2006 and 2008. The results given by this data collection contradict our initial hypothesis and reveal an important stability of the social representation. No ecological factor is brought to light. The notion of growth seems to be appropriated as a crucial and unchanging value.

\section{INDEX}

Mots-clés : représentations sociales, croissance économique

Keywords : Social representations, economic growth, sustainable development

\section{AUTEURS}

\section{HÉLÈNE BLANC}

Hélène Blanc est Maître de Conférences en Sciences Économiques, a un doctorat dans cette discipline, obtenu en 1998. Membre de l'EREID (Équipe de Recherche et d'Études Interdisciplinaires sur la Durabilité) de l'Université Européenne de Bretagne (UEB) - Université de Bretagne Occidentale et enseignante à l'IUT de Quimper. Ses recherches actuelles portent sur la décroissance et le développement durable, émergence d'un concept et représentations sociales de ces notions.

\section{ADELINE RAYMOND}

Adeline Raymond est maître de conférences en Psychologie sociale et du travail à l'Université de Bretagne Occidentale, UEB. Membre du Centre de recherche en psychologie, cognition et communication (CRPCC - EA 1285). Ses recherches actuelles portent sur les représentations sociales de la croissance et décroissance. Psychologie du travail : le sens accordé au travail, la résistance au changement, les modifications des attitudes et des pratiques et l'approche collective du travail. 